\title{
Asthma is a risk factor for thromboembolic disease in pregnancy
}

\section{Keywords}

asthma, pregnant, asthma control, thromboembolic disease

\begin{abstract}
Introduction

Finding the risk factors for thromboembolic (TE) disease and preventing its development in pregnant women is important. Asthma is a common chronic disease in pregnant women. The aim of the study is to find the risk factors.

Material and methods

From 2004 to $2011,55,057$ pregnant women were recruited from a Taiwan database. They were grouped into AS and non-AS groups. AS subjects were divided into three

groups. Group 1: with AS before pregnancy or during pregnancy; without AS after childbirth. Group 2: without AS before pregnancy, with AS during pregnancy or after childbirth. Group 3: with AS before, during pregnancy and after childbirth. The rate of TE (during pregnancy and 60 days after childbirth) was compared between different groups.
\end{abstract}

Results

In pregnant women, TE rate was $2.47 \%$ in AS and $0.71 \%$ in non-AS subjects. The rate was higher in AS subjects (OR 3.47 , adjust $p$ 0.005). In group 2, the rate was $3.97 \%$, higher than that in non-AS subjects (OR 5.44, p 0.013). The TE rate in groups 1 and 3 was similar to non-AS subjects.

Conclusions

The rate of TE increases if pregnant women have AS. Physicians need to be alert to the occurrence of TE as early as possible in pregnant women with AS. If AS was re-appeared or worsened during pregnancy (group 2), the rate of TE increased. Pregnant women and physicians should prevent the reappear of AS or control AS properly during pregnancy to prevent the occurrence of TE.

\section{Explanation letter}

Dear Editor,

The revised manuscript entitled "Asthma is a risk factor for thromboembolic disease in pregnancy" has been revised in response to reviewer's suggestions. The changes within the revised manuscript have been highlighted by mark on red.

Two papers related to this topic published in the Archives of Medical Science in last 2-3 years were cited (reference 22 and 23).

I hope this paper is suitable for "Archives of Medical Science". I deeply appreciate your consideration of the manuscript, and I look forward to receiving comments from the reviewers. If you have any queries, please don't hesitate to contact me at the address below.

Thank you and best regards.

Yours sincerely

Corresponding author:

Name: Min-Sho Ku

E-mail: a129184@yahoo.com.tw

point to point response.docx 


\section{Asthma is a risk factor for thromboembolic disease in pregnancy}

\section{ABSTRACT}

Introduction: Finding the risk factors for thromboembolic (TE) disease and preventing its development in pregnant women is important. Asthma is a common chronic disease in pregnant women. The aim of the study is to find the risk factors.

Material and methods: From 2004 to 2011, 55,057 pregnant women were recruited from a Taiwan database. They were grouped into AS and non-AS groups. AS subjects were divided into three groups. Group 1: with AS before pregnancy or during pregnancy; without AS after childbirth.

Group 2: without AS before pregnancy, with AS during pregnancy or after childbirth. Group 3: with AS before, during pregnancy and after childbirth. The rate of TE (during pregnancy and 60 days after childbirth) was compared between different groups.

Results: In pregnant women, TE rate was $2.47 \%$ in AS and $0.71 \%$ in non-AS subjects. The rate was higher in AS subjects (OR 3.47, adjust p 0.005). In group 2, the rate was 3.97\%o, higher than that in non-AS subjects (OR 5.44, p 0.013). The TE rate in groups 1 and 3 was similar to non-AS subjects.

Conclusions: The rate of TE increases if pregnant women have AS. Physicians need to be alert to the occurrence of TE as early as possible in pregnant women with AS. If AS was re-appeared or worsened during pregnancy (group 2), the rate of TE increased. Pregnant women and physicians should prevent the re-appear of AS or control AS properly during pregnancy to prevent the 
occurrence of TE.

Key Words: thromboembolic disease, asthma, pregnant, asthma control 


\section{Introduction}

Compared to non-pregnant women, there is an increased risk of thromboembolism (TE) in pregnant women [1-2]. TE, including pulmonary embolism (PE) noted during pregnancy increases the mortality and morbidity of mother and fetus [3]. The symptoms and signs of TE and PE are non-specific which causes a delayed or missed diagnosis [4]. Therefore, if we can find as many risk factors of TE as possible, we can prevent a delayed and missed diagnosis.

Through different mechanisms, AS is associated with a procoagulant state [5]. The increased risk of deep vein thrombosis and PE have was also been noted [6]. AS is also a common chronic disease in pregnant women, and the rates have increased in recent years [7]. However, the association between pregnant women with AS and TE has not been studied yet.

A study indicated that one-third of AS patients had reappear or worse of AS symptoms due to pregnancy, one-third had AS remission, and the remaining $1 / 3$ of patients had no significant changes [8]. Those who experienced remission demonstrated their AS was well controlled during pregnancy. Those whose symptoms reappear or worsened showed their AS was poorly controlled [7,9]. If AS is a risk factor of TE, does well controlled AS decrease the rate of TE in pregnant women? Does poor control of AS increase the rate of TE in pregnant women? These speculations have not been studied before.

Through a large, population-based, longitudinal database in Taiwan, we aimed to find the association between AS, pregnancy and TE. By comparing the rate of TE between non-AS and AS subjects, we aimed to understand if well controlled/remission or poorly controlled/reappear of AS 
would increase or decrease the occurrence of TE in pregnant women.

\section{Materials and Methods}

\section{Database and data collection}

A representative sample of 1 million participants from the National Health Insurance Research Database was provided by Taiwan's National Health Research Institutes [10-11], and this sample served as our data source. Information on basic patient demographics and medical care received, including outpatient, inpatient claims and childbirth was gathered. The study flowchart is presented in Fig. 1. The International Classification of Diseases, 9th Revision, Clinical Modification (ICD-9-CM) was used to identify diagnoses of diseases in the claims data. The ICD-9-CM code used in our study is presented in Table 1. The institutional review board of Chung Shan Medical University Hospital, Taichung, Taiwan, approved this study (ethics approval number: CS2-20161).

\section{Recruitment and grouping of subjects}

From 2004 to 2011, 55,057 pregnant women were selected for the study. To prevent the complex conditions of patients that would influence the results of the study, those with the diagnosis of malignancy, major illnesses such as chronic psychosis, paralysis or severe trauma before childbirth were excluded from the study. The claim data of these subjects, 3 years before and 2 years after childbirth were gathered for the study. Those with and without the diagnosis of AS during the 5 years were grouped into the asthma group and non- asthma group. The criteria of AS subjects was: at least two diagnosis of AS; or at least one diagnosis was made by thoracic, allergic or immunologic doctor; or ever use steroid, anti-leukotriene or other drugs to treat AS; or at least 
one admission or emergency department visit with the diagnosis of AS.

These AS subjects were further divided into three groups. Group 1: with AS before pregnancy or during pregnancy; without AS after childbirth. Group 2: without AS before pregnancy, with AS during pregnancy or after childbirth. Group 3: with AS before, during pregnancy and after childbirth. The criterion for "with AS before pregnancy" was at least one diagnosis of AS within 3 years before to 270 days before childbirth. The criterion for "with AS during pregnancy" was at least one diagnosis of AS 270 days before childbirth to childbirth day. The criterion for "with As after childbirth" was: at least one diagnosis of AS after childbirth. The rate of AS episodes and other allergic disease (allergic rhinitis, allergic conjunctivitis, atopic dermatitis) in different AS groups were also counted.

\section{Rate of TE}

TE diseases selected in our study are presented in Table 1.Those with TE diagnosis was counted as "with TE". The criteria of "with TE" were: had one or more TE diagnoses during pregnancy and 60 days after childbirth. To make the diagnosis reliable, they were diagnosed by cardiac, thoracic or surgical doctor; or received anti-coagulant or surgical therapy; or received examination for TE such as angiography, deplex ultrasonography, or others during pregnant and 6 months after childbirth. The rates of TE were compared between AS and non-AS subjects. The rates of TE in the three groups were also compared to that of the non-AS group. In those who underwent cesarean delivery, the rates of TE were also compared between groups. According to the diagnosis of obesity or not, these subjects were grouped to (1) non-AS, non-obesity (2) non-AS, obesity (3) AS, non-obesity (4) 
AS, obesity groups. The rates of TE in the four groups were also compared.

\section{Rate of venous complications}

Those with "venous complications in pregnancy and puerperium" were counted as "with venous complications". The criteria of "with venous complications" were: had one or more venous complications diagnoses during pregnancy and 60 days after childbirth. The rates of venous complications in the three AS groups were compared to that of the non-AS group.

\section{Determining the confounding factors}

Royal College of Obstetricians \& Gynecologists (RCOG) [12] summarized the risk factors for TE during pregnancy and puerperium. These include: risk factors related to general conditions or medical conditions, obstetrical and transient factors. According to the guideline, birth type (cesarean versus vaginal delivery), mother's age (less than versus equal or more than 35 years old), medical co-morbidities, pregnancy characteristics and delivery complications were viewed as the confounding factors in the study. Socioeconomic status, urbanization that might influence characteristics of pregnancy and delivery were also considered as the confounding factors. Socioeconomic status was defined according to occupation, which was grouped as middle to highsocioeconomic status (teacher or public official, company employee) and low- socioeconomic status (other, peasants or fisherman, low income or no fixed job). Based on Liu's report [13], urbanization levels were grouped into seven levels: levels 1-2 for urban residents, and levels 3-7 for country residents.

\section{Statistical analysis}


All statistical analyses were performed using SAS software version 9.1 (SAS Institute Inc., Carey, NC), and PASW Statistics 18 (IBM, Armonk, NY, USA). The chi-square test was used to compare the rate of TE between groups. Multivariate logistic regression analyses were used for adjusting for the confounding factors. Two-sided $p$ values of $<0.05$ were defined as significant.

\section{Results}

\section{Study flowchart and demographic data}

The study flowchart is presented in Figure 1. The demographic data of AS and non-AS groups is presented in Table 2. A total of 55,057 women were recruited, 3,231 (5.87\%) of them were diagnosed with AS. The rate of cesarean delivery, medical co-morbidities, pregnancy characteristics, delivery complications were higher in AS group.

\section{Rate of TE and venous complication between AS and non-AS pregnant women}

The rate of TE between AS and non-AS pregnant women is presented in Table 2. The rate of TE in the AS group was 2.47 per 1000 subjects (2.47\%), and the rate of TE in the non-AS group was 0.71 per 1000 subjects $(0.71 \%)$. The $p$ value was 0.001 , Odds Ratio (OR) was $3.47 ; 95 \%$ confidence interval (CI) was 1.62 7.47. After adjusting for the confounding factors, the $p$ value was 0.005. The rate of venous complications in the AS group was $7.12 \%$, and the rate in the non-AS group was 6.85\%o. The $p$ value was 0.858 (OR 1.04; 95\% CI 0.68 1.59). After adjusting for the confounding factors, the $p$ value was 0.700 .

\section{Rate of asthma episodes and other allergic disease in different AS groups}

Before pregnancy, mean asthma diagnosis times in group 1, 2 and 3 were 2.21, 0, and 6.44 
times respectively. During pregnancy, the times were $0.39,0.14$, and 1.87 times respectively. After childbirth, the times were $0,1.87$, and 4.79 times respectively. AS diagnosis times were all higher in group 3. Before pregnancy, the rate of other allergic disease in no-AS subjects was $27.73 \%$; and $41.67 \%$ in AS, group 2. The rate was higher in group $2(\mathrm{p}=0.000)$. During pregnancy, the rate of other allergic disease in no-AS subjects was $12.04 \%$; and $22.85 \%$ in AS, group 2. The rate was still higher in group $2(\mathrm{p}=0.000)$.

\section{Rate of TE between the three AS groups and non-AS subjects}

The results of TE rates in different AS groups are presented in Table 3. In group 2 (without AS before pregnancy, with AS during pregnancy or after childbirth), the rate of TE was $3.97 \%$. The rate of TE in the non-AS group was $0.71 \%$. Group 2 had higher rates of TE than the non-AS subjects (OR 5.44, 95\% CI 1.67 17.68, adjust p-value $=0.013$ ). The rate of TE in group 1 (with AS before pregnancy or during pregnancy; without AS after childbirth) and group 3 (with AS before, during pregnancy and after childbirth) was not higher than the non-AS subjects.

\section{Rate of venous complications between the three AS groups and non-AS subjects}

The results of venous complication rates in different AS groups are presented in Table 4. In group 3, the rate of venous complications was $15.98 \%$. The rate of venous complications in the non-AS subjects was $6.85 \%$. Group 3 had higher venous complication rates than the non-AS subjects (OR 2.36, 95\% CI 1.11 5.01, adjust p-value $=0.043$ ). The rate of venous complications in group 1 and group 2 was not higher than the non-AS subjects. 


\section{non-AS subjects}

For those who underwent cesarean delivery, the rates of TE in different AS groups are presented in Table 5. In group 2, the rate of TE was $6.45 \%$. The rate of TE in the non-AS subjects was $1.23 \%$. Group 2 had higher TE rates than the non-AS subjects (OR 5.27, 95\% CI 1.24 22.46, adjusted p-value $=0.046$ ). The rate of TE in group 1 and group 3 was similar to the non-AS subjects.

\section{Rate of TE in obesity and non-obesity subjects}

The rate of TE in non-AS, non-obesity subjects was $0.66 \%$. Compare to them, the rates of TE were higher in non-AS, obesity (6.45\%, adjust p-value $=0.005$, OR 3.33); AS, non-obesity (2.20\%o, adjust $p$-value $=0.007$, OR 9.80) and AS, obesity subjects $(17.54 \%$, adjust $p$-value $=0.011$, OR 26.96).

\section{Discussion}

In this population based, retrospective cohort study, we found an association between AS and thromoembolic (TE) disease in pregnant women. In pregnant AS subjects, the rate of TE (during pregnancy and 60 days after childbirth) was higher compared to non-AS subjects. Studies indicate that maternal AS increases the adverse complications in fetuses such as small for gestational age, congenital malformations and increased perinatal mortality. Maternal morbidities, such as preeclampsia, gestational hypertension, gestational diabetes and urinary tract infections also increase [14]. However, whether AS increases during pregnancy or not has not been studied before. In this study, we found an association between AS and TE in pregnant women. The rate of TE in pregnant women increased if they had asthma. 
Obesity is a risk factor of TE during and after pregnancy [12]. Our study results showed that, compare to non-obesity subjects, the rates of TE were higher in those with obesity, especially in AS plus obesity subjects (OR 26.96). It reminds doctors and pregnant women to notice the happen of TE if pregnant women had both AS and obesity.

Normal pregnancy is associated with Th2 (T helper cells 2) skewing. Th2 skewing is required for a successful pregnancy. Th2 cells are associated with the production of immunoglobulin $\mathrm{E}(\operatorname{IgE})$ antibodies and allergic disease including asthma [15]. Through the interaction with mast cells, $\operatorname{IgE}$ exert some prothrombotic and antifibrinolytic activities. This mechanism and other mechanisms could explain our finding that AS was associated with the increase of TE in pregnant women [16].

A study has shown that half of the women with AS discontinued therapy thus diminishing the control of the disease, mostly due to inadequate education and fear of adverse events [17].

This is sometimes encouraged by insufficiently educated physicians. Inadequate therapy, or discontinued treatment of AS during pregnancy may result in worsening of AS. The worsening of AS symptoms increases the rate of adverse events, as mentioned above, both in mother and fetus

\section{$[14,17]$.}

A study has indicated that there is no research which demonstrates that asthma can be induced due to pregnancy. Instead, pregnancy can cause asthma symptoms to worsen [18]. Therefore, we speculated that, those without AS before pregnancy but with AS after pregnancy (group 2) were those whose AS might be well controlled and not attack before pregnancy. However, their AS was re-appeared and worse during pregnancy. Poor AS control during pregnancy might be the reason. 
Those with AS before, during pregnancy but without after (group 1) were those with AS remission during pregnancy. Well AS control during pregnancy or other factors might be the reason of the remission. Our study results remind pregnant women and physicians to control or prevent AS properly during pregnancy to prevent the occurrence of TE. Although there is no AS before pregnancy, the rate of other allergic disease before pregnancy was still higher in group 2, compare to the controls. This remind us that, the happen of other allergic disease before pregnancy is a warning of TE later, despite the happen of AS or not.

A study found that higher concentrations of $\operatorname{IgE}$ might predict the onset of severe complications of pulmonary embolism [16]. Increased IgE levels, an indicator of severity of AS, increases the rates of TE. Another study demonstrated that compared to a stable stage, during asthma exacerbation, airway and systemic inflammations increased. This results in the activation of the systemic coagulation pathway [19]. Asthma group 3 had the highest AS attack times. It means that their AS was most severe. However, their TE rates were not higher. Therefore, we speculated that, AS re-appear or worse during pregnancy, rather than its severity, was the cause of the increase of TE. However, more studies are necessary.

Vasoactive compounds (histamine, tryptase and other cytokines) released from mast cells play an important role in the development of varicose veins [20]. When allergen-IgE complex binds to mast cells, the cells are activated, and release histamine and other cytokines. Elevated histamine and cytokine levels then induce the development of varicose veins. Therefore, we speculated that venous complications including varicose veins might play a role in the AS, TE and pregnancy 
association. In our study, the rate of venous complications was higher in group 3. However, the rate of TE did not increase in group 3. Therefore, although venous complications accompany pregnancy, the increase of venous complications was not the cause of the increase of TE in pregnant women with AS.

There are limitations in our study. First, there is no laboratory data to evaluate the clinical condition of AS and TE. Second, there is no radiological data. Radiological data is important to evaluate the profile of TE. Third, PE is one of the most severe diseases in TE. Evaluating the association between AS and PE in pregnant women is important. However, because patient numbers of PE was too few, it could not be calculated. Further investigation with the recruitment of more subjects is necessary to find their association. Fourth, other factors such as maternal smoking [21], vitamin D [22] and others might influence the development of asthma, health of pregnancy and TE. Future studies are necessary to find their influence.

\section{Conclusion}

Through this population based, retrospective cohort study, we first found an association between AS and TE in pregnant women. Physicians need to pay more attention to detect TE as early as possible in pregnant women with AS, especially those combined obesity and overweight. In those without AS attacks before pregnancy, but AS re-appear or worse during pregnancy, the rate of TE increased more. This reminds pregnant women and physicians to prevent the reappear of AS or control AS properly during pregnancy. Future study, including case-control studies, more clinical 
data and biological research, are necessary to fully understand the association between AS and TE in pregnant women. 


\section{References}

1.Kane EV, Calderwood C, Dobbie R, Morris C, Roman E, Greer IA. A population-based study of venous thrombosis in pregnancy in Scotland 1980-2005. Eur J Obstet Gynecol Reprod Biol. 2013; 169: 223-9.

2. Marik PE, Plante LA. Venous thromboembolic disease and pregnancy. N Engl J Med. 2008; 359: 2025-33.

3. Greer IA. CLINICAL PRACTICE. Pregnancy complicated by venous thrombosis. N Engl J Med. $2015 ; 373: 540-7$.

4. Ozsu S, Oztuna F, Bulbul Y, Topbas M, Ozlu T, Kosucu P, Ozsu A. The role of risk factors in delayed diagnosis of pulmonary embolism. Am Emerg Med 2011; 29:2 6-32.

5. de Boer JD, Majoor CJ, van 't Veer C, Bel EH, van der Poll T, Boer JDd. Asthma and coagulation. Blood 2012; 119: 3236-44.

6. Majoor CJ, Kamphuisen PW, Zwinderman AH, et al. Risk of deep vein thrombosis and pulmonary embolism in asthma. Eur Respir J 2013; 42: 655-61.

7. Wang H, Li N, Huang H. Asthma in Pregnancy: Pathophysiology, Diagnosis, Whole-Course Management, and Medication Safety. Can Respir J 2020; 9046842.

8. Schatz M, Harden K, Forsythe A, Chilingar L, Hoffman C, Sperling W, Zeiger RS. The course of asthma during pregnancy, post partum, and with successive pregnancies: a prospective analysis. $\mathrm{J}$ Allergy Clin Immunol 1988; 81: 509-17.

9. Bonham CA, Patterson KC, Strek ME. Asthma Outcomes and Management During Pregnancy. 
Chest 2018; 153: 515-27.

10. Department of health, E. Y. (1998) National Health Insurance Evaluation and Projections.

Taiwan: 1998, DoH, 1.

11. Cheng TM: Taiwan's new national health insurance program: genesis and experience so far.

Health Aff 2003; 22: 61-76.

12. Royal Colledge of Obstetricians \& Gynecologists, Reducing the Risk of Venous

Thromboembolism during Pregnancy and the Puerperium, Greentop Guideline No. 37a, 2015.

13. CY Liu, YT Hung, YL Chuang, YJ Chen, WS Weng, JS Liu. Incorporating development stratification of Taiwan townships into sampling design of large scale health interview survey. J

Health Manage 2006, 14: 1-22.

14. Hodyl NA, Stark MJ, Scheil W, Grzeskowiak LE, Clifton VL. Perinatal outcomes following maternal asthma and cigarette smoking during pregnancy. Eur Respir J 2014; 42: 704-16.

15. McFadden JP, Thyssen JP, Basketter DA, Puangpet P, Kimber I. T helper cell 2 immune skewing in pregnancy/early life: chemical exposure and the development of atopic disease and allergy. Br J Dermatol 2015; 172: 584-91.

16. Chung WS, Lin CL, Ho FM, Li RY, Sung FC, Kao CH, Yeh JJ. Asthma increases pulmonary thromboembolism risk: a nationwide population cohort study. Eur Respir J 2014; 43: 801-7.

17. Labor S, Dalbello Tir AM, Plavec D, Juric I, Roglic M, Pavkov Vukelic J, Labor M. What is safe enough - asthma in pregnancy - a review of current literature and recommendations. Asthma Res Pract 2018; 4: 11. 
18. Pregnancy and Asthma - ACAAI Patient.

https://acaai.org/asthma/asthma-101/who-gets-asthma/pregnancy-and-asthma/.

19. Manuyakorn W, Mairiang D, Sirachainan N, Kadegasem P, Kamchaisatian W, Benjaponpitak S, Chuansumrit A. A. Blood Coagulation and Asthma Exacerbation in Children. Int Arch Allergy Immunol 2016; 170: 75-83.

20. Haviarova Z, Weismann P, Pavlikova D, Durdik S, Kovac P, Stvrtinova V, Mraz P. Mast cell infiltration in the wall of varicose veins. Acta Histochem 2002; 104: 357-60.

21. Wojtyla C, Wojtyla-Buciora P, Ciebiera M, Orzechowski S, Wojtyla A. The effect of active and passive maternal smoking before and during pregnancy on neonatal weight at birth. Arch Med Sci 2018;17: 352-60.

22. Ciebiera M, Wojtyla C, Lukaszuk K, Zgliczynska M, Zareba K, Rawski W, Jakiel G The role of vitamin D in perinatology. An up-to-date review. Arch Med Sci 2019; 17: 992-1005. 


\section{Asthma is a risk factor for thromboembolic disease in pregnancy}

\section{ABSTRACT}

Introduction: Finding the risk factors for thromboembolic (TE) disease and preventing its development in pregnant women is important. Asthma is a common chronic disease in pregnant women. The aim of the study is to find the risk factors.

Material and methods: From 2004 to 2011, 55,057 pregnant women were recruited from a Taiwan database. They were grouped into AS and non-AS groups. AS subjects were divided into three groups. Group 1: with AS before pregnancy or during pregnancy; without AS after childbirth.

Group 2: without AS before pregnancy, with AS during pregnancy or after childbirth. Group 3: with AS before, during pregnancy and after childbirth. The rate of TE (during pregnancy and 60 days after childbirth) was compared between different groups.

Results: In pregnant women, TE rate was $2.47 \%$ in AS and $0.71 \%$ in non-AS subjects. The rate was higher in AS subjects (OR 3.47, adjust p 0.005). In group 2, the rate was 3.97\%, higher than that in non-AS subjects (OR 5.44, p 0.013). The TE rate in groups 1 and 3 was similar to non-AS subjects.

Conclusions: The rate of TE increases if pregnant women have AS. Physicians need to be alert to the occurrence of TE as early as possible in pregnant women with AS. If AS was re-appeared or worsened during pregnancy (group 2), the rate of TE increased. Pregnant women and physicians should prevent the re-appear of AS or control AS properly during pregnancy to prevent the 
occurrence of TE.

Key Words: thromboembolic disease, asthma, pregnant, asthma control 


\section{Introduction}

Compared to non-pregnant women, there is an increased risk of thromboembolism (TE) in pregnant women [1-2]. TE, including pulmonary embolism (PE) noted during pregnancy increases the mortality and morbidity of mother and fetus [3]. The symptoms and signs of TE and PE are non-specific which causes a delayed or missed diagnosis [4]. Therefore, if we can find as many risk factors of TE as possible, we can prevent a delayed and missed diagnosis.

Through different mechanisms, AS is associated with a procoagulant state [5]. The increased risk of deep vein thrombosis and PE have was also been noted [6]. AS is also a common chronic disease in pregnant women, and the rates have increased in recent years [7]. However, the association between pregnant women with AS and TE has not been studied yet.

A study indicated that one-third of AS patients had reappear or worse of AS symptoms due to pregnancy, one-third had AS remission, and the remaining $1 / 3$ of patients had no significant changes [8]. Those who experienced remission demonstrated their AS was well controlled during pregnancy. Those whose symptoms reappear or worsened showed their AS was poorly controlled [7,9]. If AS is a risk factor of TE, does well controlled AS decrease the rate of TE in pregnant women? Does poor control of AS increase the rate of TE in pregnant women? These speculations have not been studied before.

Through a large, population-based, longitudinal database in Taiwan, we aimed to find the association between AS, pregnancy and TE. By comparing the rate of TE between non-AS and AS subjects, we aimed to understand if well controlled/remission or poorly controlled/reappear of AS 
would increase or decrease the occurrence of TE in pregnant women.

\section{Materials and Methods}

\section{Database and data collection}

A representative sample of 1 million participants from the National Health Insurance Research Database was provided by Taiwan's National Health Research Institutes [10-11], and this sample served as our data source. Information on basic patient demographics and medical care received, including outpatient, inpatient claims and childbirth was gathered. The study flowchart is presented in Fig. 1. The International Classification of Diseases, 9th Revision, Clinical Modification (ICD-9-CM) was used to identify diagnoses of diseases in the claims data. The ICD-9-CM code used in our study is presented in Table 1. The institutional review board of Chung Shan Medical University Hospital, Taichung, Taiwan, approved this study (ethics approval number: CS2-20161).

\section{Recruitment and grouping of subjects}

From 2004 to 2011, 55,057 pregnant women were selected for the study. To prevent the complex conditions of patients that would influence the results of the study, those with the diagnosis of malignancy, major illnesses such as chronic psychosis, paralysis or severe trauma before childbirth were excluded from the study. The claim data of these subjects, 3 years before and 2 years after childbirth were gathered for the study. Those with and without the diagnosis of AS during the 5 years were grouped into the asthma group and non- asthma group. The criteria of AS subjects was: at least two diagnosis of AS; or at least one diagnosis was made by thoracic, allergic 
one admission or emergency department visit with the diagnosis of AS.

These AS subjects were further divided into three groups. Group 1: with AS before pregnancy or during pregnancy; without AS after childbirth. Group 2: without AS before pregnancy, with AS during pregnancy or after childbirth. Group 3: with AS before, during pregnancy and after childbirth. The criterion for "with AS before pregnancy" was at least one diagnosis of AS within 3 years before to 270 days before childbirth. The criterion for "with AS during pregnancy" was at least one diagnosis of AS 270 days before childbirth to childbirth day. The criterion for "with As after childbirth" was: at least one diagnosis of AS after childbirth. The rate of AS episodes and other allergic disease (allergic rhinitis, allergic conjunctivitis, atopic dermatitis) in different AS groups were also counted.

\section{Rate of TE}

TE diseases selected in our study are presented in Table 1.Those with TE diagnosis was counted as "with TE". The criteria of "with TE" were: had one or more TE diagnoses during pregnancy and 60 days after childbirth. To make the diagnosis reliable, they were diagnosed by cardiac, thoracic or surgical doctor; or received anti-coagulant or surgical therapy; or received examination for TE such as angiography, deplex ultrasonography, or others during pregnant and 6 months after childbirth.

The rates of TE were compared between AS and non-AS subjects. The rates of TE in the three groups were also compared to that of the non-AS group. In those who underwent cesarean delivery, the rates of TE were also compared between groups. According to the diagnosis of obesity or not, these subjects were grouped to (1) non-AS, non-obesity (2) non-AS, obesity (3) AS, non-obesity (4) 
AS, obesity groups. The rates of TE in the four groups were also compared.

\section{Rate of venous complications}

Those with "venous complications in pregnancy and puerperium" were counted as "with venous complications". The criteria of "with venous complications" were: had one or more venous complications diagnoses during pregnancy and 60 days after childbirth. The rates of venous complications in the three AS groups were compared to that of the non-AS group.

\section{Determining the confounding factors}

Royal College of Obstetricians \& Gynecologists (RCOG) [12] summarized the risk factors for TE during pregnancy and puerperium. These include: risk factors related to general conditions or medical conditions, obstetrical and transient factors. According to the guideline, birth type (cesarean versus vaginal delivery), mother's age (less than versus equal or more than 35 years old), medical co-morbidities, pregnancy characteristics and delivery complications were viewed as the confounding factors in the study. Socioeconomic status, urbanization that might influence characteristics of pregnancy and delivery were also considered as the confounding factors. Socioeconomic status was defined according to occupation, which was grouped as middle to highsocioeconomic status (teacher or public official, company employee) and low-socioeconomic status (other, peasants or fisherman, low income or no fixed job). Based on Liu's report [13], urbanization levels were grouped into seven levels: levels 1-2 for urban residents, and levels 3-7 for country residents.

\section{Statistical analysis}


All statistical analyses were performed using SAS software version 9.1 (SAS Institute Inc., Carey, NC), and PASW Statistics 18 (IBM, Armonk, NY, USA). The chi-square test was used to compare the rate of TE between groups. Multivariate logistic regression analyses were used for adjusting for the confounding factors. Two-sided $p$ values of $<0.05$ were defined as significant.

\section{Results}

\section{Study flowchart and demographic data}

The study flowchart is presented in Figure 1. The demographic data of AS and non-AS groups is presented in Table 2. A total of 55,057 women were recruited, 3,231 (5.87\%) of them were diagnosed with AS. The rate of cesarean delivery, medical co-morbidities, pregnancy characteristics, delivery complications were higher in AS group.

\section{Rate of TE and venous complication between AS and non-AS pregnant women}

The rate of TE between AS and non-AS pregnant women is presented in Table 2. The rate of TE in the AS group was 2.47 per 1000 subjects (2.47\%), and the rate of TE in the non-AS group was 0.71 per 1000 subjects $(0.71 \%$ ). The $p$ value was 0.001 , Odds Ratio (OR) was $3.47 ; 95 \%$ confidence interval (CI) was 1.62 7.47. After adjusting for the confounding factors, the $p$ value was 0.005. The rate of venous complications in the AS group was 7.12\%, and the rate in the non-AS group was 6.85\%. The $p$ value was 0.858 (OR 1.04; 95\% CI 0.68 1.59). After adjusting for the confounding factors, the $p$ value was 0.700 .

Rate of asthma episodes and other allergic disease in different AS groups 
times respectively. During pregnancy, the times were $0.39,0.14$, and 1.87 times respectively. After childbirth, the times were $0,1.87$, and 4.79 times respectively. AS diagnosis times were all higher in group 3. Before pregnancy, the rate of other allergic disease in no-AS subjects was $27.73 \%$; and $41.67 \%$ in AS, group 2. The rate was higher in group $2(\mathrm{p}=0.000)$. During pregnancy, the rate of other allergic disease in no-AS subjects was $12.04 \%$; and $22.85 \%$ in AS, group 2. The rate was still higher in group $2(\mathrm{p}=0.000)$.

Rate of TE between the three AS groups and non-AS subjects

The results of TE rates in different AS groups are presented in Table 3. In group 2 (without AS before pregnancy, with AS during pregnancy or after childbirth), the rate of TE was 3.97\%o. The rate of TE in the non-AS group was $0.71 \%$. Group 2 had higher rates of TE than the non-AS subjects (OR 5.44, 95\% CI 1.67 17.68, adjust $\mathrm{p}$-value $=0.013)$. The rate of TE in group 1 (with AS before pregnancy or during pregnancy; without AS after childbirth) and group 3 (with AS before, during pregnancy and after childbirth) was not higher than the non-AS subjects.

Rate of venous complications between the three AS groups and non-AS subjects

The results of venous complication rates in different AS groups are presented in Table 4. In group 3, the rate of venous complications was $15.98 \%$. The rate of venous complications in the non-AS subjects was $6.85 \%$. Group 3 had higher venous complication rates than the non-AS subjects (OR 2.36, 95\% CI 1.11 5.01, adjust p-value $=0.043)$. The rate of venous complications in group 1 and group 2 was not higher than the non-AS subjects.

Rate of TE in those who underwent cesarean delivery between the three AS groups and 
non-AS subjects

For those who underwent cesarean delivery, the rates of TE in different AS groups are presented in Table 5. In group 2, the rate of TE was 6.45\%. The rate of TE in the non-AS subjects was $1.23 \%$. Group 2 had higher TE rates than the non-AS subjects (OR 5.27, 95\% CI 1.24 22.46, adjusted p-value $=0.046)$. The rate of TE in group 1 and group 3 was similar to the non-AS subjects.

Rate of TE in obesity and non-obesity subjects

The rate of TE in non-AS, non-obesity subjects was $0.66 \%$. Compare to them, the rates of TE were higher in non-AS, obesity (6.45\%, adjust p-value $=0.005$, OR 3.33); AS, non-obesity (2.20\%, adjust $\mathrm{p}$-value $=0.007, \mathrm{OR} 9.80)$ and AS, obesity subjects $(17.54 \%$, adjust p-value $=0.011, \mathrm{OR}$ 26.96).

\section{Discussion}

In this population based, retrospective cohort study, we found an association between AS and thromoembolic (TE) disease in pregnant women. In pregnant AS subjects, the rate of TE (during pregnancy and 60 days after childbirth) was higher compared to non-AS subjects. Studies indicate that maternal AS increases the adverse complications in fetuses such as small for gestational age, congenital malformations and increased perinatal mortality. Maternal morbidities, such as preeclampsia, gestational hypertension, gestational diabetes and urinary tract infections also increase [14]. However, whether AS increases during pregnancy or not has not been studied before. In this study, we found an association between AS and TE in pregnant women. The rate of TE in pregnant women increased if they had asthma. 
Obesity is a risk factor of TE during and after pregnancy [12]. Our study results showed that, compare to non-obesity subjects, the rates of TE were higher in those with obesity, especially in AS plus obesity subjects (OR 26.96). It reminds doctors and pregnant women to notice the happen of TE if pregnant women had both AS and obesity.

Normal pregnancy is associated with Th2 (T helper cells 2) skewing. Th2 skewing is required for a successful pregnancy. Th2 cells are associated with the production of immunoglobulin $\mathrm{E}(\operatorname{IgE})$ antibodies and allergic disease including asthma [15]. Through the interaction with mast cells, IgE exert some prothrombotic and antifibrinolytic activities. This mechanism and other mechanisms could explain our finding that AS was associated with the increase of TE in pregnant women [16].

A study has shown that half of the women with AS discontinued therapy thus diminishing the control of the disease, mostly due to inadequate education and fear of adverse events [17].

This is sometimes encouraged by insufficiently educated physicians. Inadequate therapy, or discontinued treatment of AS during pregnancy may result in worsening of AS. The worsening of AS symptoms increases the rate of adverse events, as mentioned above, both in mother and fetus $[14,17]$.

A study has indicated that there is no research which demonstrates that asthma can be induced due to pregnancy. Instead, pregnancy can cause asthma symptoms to worsen [18]. Therefore, we speculated that, those without AS before pregnancy but with AS after pregnancy (group 2) were those whose AS might be well controlled and not attack before pregnancy. However, their AS was re-appeared and worse during pregnancy. Poor AS control during pregnancy might be the reason. 
Those with AS before, during pregnancy but without after (group 1) were those with AS remission during pregnancy. Well AS control during pregnancy or other factors might be the reason of the remission. Our study results remind pregnant women and physicians to control or prevent AS properly during pregnancy to prevent the occurrence of TE. Although there is no AS before pregnancy, the rate of other allergic disease before pregnancy was still higher in group 2, compare to the controls. This remind us that, the happen of other allergic disease before pregnancy is a warning of TE later, despite the happen of AS or not.

A study found that higher concentrations of $\operatorname{IgE}$ might predict the onset of severe complications of pulmonary embolism [16]. Increased IgE levels, an indicator of severity of AS, increases the rates of TE. Another study demonstrated that compared to a stable stage, during asthma exacerbation, airway and systemic inflammations increased. This results in the activation of the systemic coagulation pathway [19]. Asthma group 3 had the highest AS attack times. It means that their AS was most severe. However, their TE rates were not higher. Therefore, we speculated that, AS re-appear or worse during pregnancy, rather than its severity, was the cause of the increase of TE. However, more studies are necessary.

Vasoactive compounds (histamine, tryptase and other cytokines) released from mast cells play an important role in the development of varicose veins [20]. When allergen-IgE complex binds to mast cells, the cells are activated, and release histamine and other cytokines. Elevated histamine and cytokine levels then induce the development of varicose veins. Therefore, we speculated that venous complications including varicose veins might play a role in the AS, TE and pregnancy 
association. In our study, the rate of venous complications was higher in group 3. However, the rate of TE did not increase in group 3. Therefore, although venous complications accompany pregnancy, the increase of venous complications was not the cause of the increase of TE in pregnant women with AS.

There are limitations in our study. First, there is no laboratory data to evaluate the clinical condition of AS and TE. Second, there is no radiological data. Radiological data is important to evaluate the profile of TE. Third, PE is one of the most severe diseases in TE. Evaluating the association between AS and PE in pregnant women is important. However, because patient numbers of PE was too few, it could not be calculated. Further investigation with the recruitment of more subjects is necessary to find their association. Fourth, other factors such as maternal smoking [21], vitamin D [22] and others might influence the development of asthma, health of pregnancy and TE. Future studies are necessary to find their influence.

\section{Conclusion}

Through this population based, retrospective cohort study, we first found an association between AS and TE in pregnant women. Physicians need to pay more attention to detect TE as early as possible in pregnant women with AS, especially those combined obesity and overweight. In those without AS attacks before pregnancy, but AS re-appear or worse during pregnancy, the rate of TE increased more. This reminds pregnant women and physicians to prevent the reappear of AS or control AS properly during pregnancy. Future study, including case-control studies, more clinical 
data and biological research, are necessary to fully understand the association between AS and TE in pregnant women. 


\section{References}

1.Kane EV, Calderwood C, Dobbie R, Morris C, Roman E, Greer IA. A population-based study of venous thrombosis in pregnancy in Scotland 1980-2005. Eur J Obstet Gynecol Reprod Biol. 2013; 169: 223-9.

2. Marik PE, Plante LA. Venous thromboembolic disease and pregnancy. N Engl J Med. 2008; 359: 2025-33.

3. Greer IA. CLINICAL PRACTICE. Pregnancy complicated by venous thrombosis. N Engl J Med. $2015 ; 373: 540-7$.

4. Ozsu S, Oztuna F, Bulbul Y, Topbas M, Ozlu T, Kosucu P, Ozsu A. The role of risk factors in delayed diagnosis of pulmonary embolism. Am Emerg Med 2011; 29:2 6-32.

5. de Boer JD, Majoor CJ, van 't Veer C, Bel EH, van der Poll T, Boer JDd. Asthma and coagulation. Blood 2012; 119: 3236-44.

6. Majoor CJ, Kamphuisen PW, Zwinderman AH, et al. Risk of deep vein thrombosis and pulmonary embolism in asthma. Eur Respir J 2013; 42: 655-61.

7. Wang H, Li N, Huang H. Asthma in Pregnancy: Pathophysiology, Diagnosis, Whole-Course Management, and Medication Safety. Can Respir J 2020; 9046842.

8. Schatz M, Harden K, Forsythe A, Chilingar L, Hoffman C, Sperling W, Zeiger RS. The course of asthma during pregnancy, post partum, and with successive pregnancies: a prospective analysis. J Allergy Clin Immunol 1988; 81: 509-17.

9. Bonham CA, Patterson KC, Strek ME. Asthma Outcomes and Management During Pregnancy. 
Chest 2018; 153: 515-27.

10. Department of health, E. Y. (1998) National Health Insurance Evaluation and Projections.

Taiwan: 1998, DoH, 1.

11. Cheng TM: Taiwan's new national health insurance program: genesis and experience so far.

Health Aff 2003; 22: 61-76.

12. Royal Colledge of Obstetricians \& Gynecologists, Reducing the Risk of Venous

Thromboembolism during Pregnancy and the Puerperium, Greentop Guideline No. 37a, 2015.

13. CY Liu, YT Hung, YL Chuang, YJ Chen, WS Weng, JS Liu. Incorporating development stratification of Taiwan townships into sampling design of large scale health interview survey. J

Health Manage 2006, 14: 1-22.

14. Hodyl NA, Stark MJ, Scheil W, Grzeskowiak LE, Clifton VL. Perinatal outcomes following maternal asthma and cigarette smoking during pregnancy. Eur Respir J 2014; 42: 704-16.

15. McFadden JP, Thyssen JP, Basketter DA, Puangpet P, Kimber I. T helper cell 2 immune skewing in pregnancy/early life: chemical exposure and the development of atopic disease and allergy. Br J Dermatol 2015; 172: 584-91.

16. Chung WS, Lin CL, Ho FM, Li RY, Sung FC, Kao CH, Yeh JJ. Asthma increases pulmonary thromboembolism risk: a nationwide population cohort study. Eur Respir J 2014; 43: 801-7.

17. Labor S, Dalbello Tir AM, Plavec D, Juric I, Roglic M, Pavkov Vukelic J, Labor M. What is safe enough - asthma in pregnancy - a review of current literature and recommendations. Asthma Res Pract 2018; 4: 11. 
18. Pregnancy and Asthma - ACAAI Patient.

https://acaai.org/asthma/asthma-101/who-gets-asthma/pregnancy-and-asthma/.

19. Manuyakorn W, Mairiang D, Sirachainan N, Kadegasem P, Kamchaisatian W, Benjaponpitak S, Chuansumrit A. A. Blood Coagulation and Asthma Exacerbation in Children. Int Arch Allergy Immunol 2016; 170: 75-83.

20. Haviarova Z, Weismann P, Pavlikova D, Durdik S, Kovac P, Stvrtinova V, Mraz P. Mast cell infiltration in the wall of varicose veins. Acta Histochem 2002; 104: 357-60.

21. Wojtyla C, Wojtyla-Buciora P, Ciebiera M, Orzechowski S, Wojtyla A. The effect of active and passive maternal smoking before and during pregnancy on neonatal weight at birth. Arch Med Sci 2018;17: 352-60.

22. Ciebiera M, Wojtyla C, Lukaszuk K, Zgliczynska M, Zareba K, Rawski W, Jakiel G The role of vitamin D in perinatology. An up-to-date review. Arch Med Sci 2019; 17: 992-1005. 
Figure 1. Flow chart

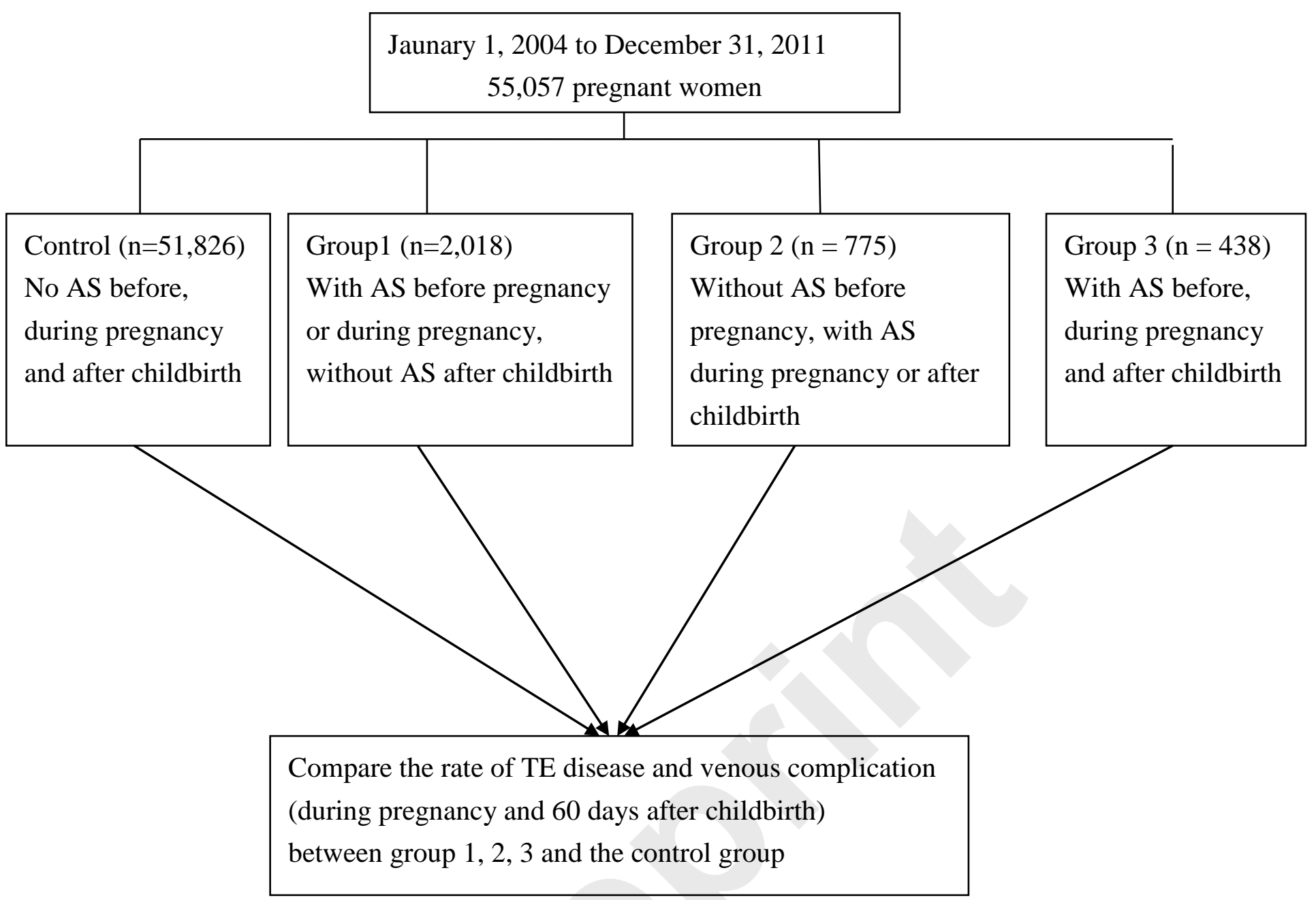


Table 1. ICD-9-CM codes used in this study.

\begin{tabular}{|c|c|}
\hline Disease & ICD-9-CM code \\
\hline Asthma & 493 \\
\hline \multicolumn{2}{|l|}{ Thromboembolism } \\
\hline TE of intracranial venous sinuses & 325 \\
\hline Pulmonary embolism and infarction & 415.1 \\
\hline TE of arteries, arterioles and capillaries & 444,445 \\
\hline Venous TE & $451,452,453$ \\
\hline Venous TE in pregnancy and puerperium & $671.2 \sim 671.5$ \\
\hline Obstetrical pulmonary embolism & $673.2,673.3,673.8$ \\
\hline \multicolumn{2}{|l|}{ Medical comorbidities } \\
\hline Type I DM with nephropathy & $250.41,250.43$ \\
\hline Chronic heart disease & $393-398,424-429$ \\
\hline Systemic lupus erythematosus & $695.4,710.0$ \\
\hline Inflammatory bowel disease & 555,556 \\
\hline Overweight and besity & 278 \\
\hline Thrombophilia & $286.53,289.81,289.82$ \\
\hline Nephrotic syndrome & 581 \\
\hline Tuberculosis & 010 018 \\
\hline Inflammatory polyarthropathy & 716.59 \\
\hline \multicolumn{2}{|l|}{ Pregnancy characteristics } \\
\hline Multiple gestation & 651 \\
\hline Preeclampsia & $642.4 \sim 642.7$ \\
\hline Pregnancy-related hypertension & $642.0 \sim 642.3,642.9$ \\
\hline \multicolumn{2}{|l|}{ Delivery complications } \\
\hline Early or threatened labor & 644 \\
\hline Postpartum hemorrhage & 666,667 \\
\hline Prolong labor & 662 \\
\hline Infection ( 7 days before $\sim 60$ days after & 590.1, 590.8, 590.9, 595.0 595.4, \\
\hline childbirth) & $\begin{array}{l}595.9,647,658.4,659.2,659.3, \\
670.0,672,675,5990,486,998.59\end{array}$ \\
\hline $\begin{array}{l}\text { Venous complications in pregnancy and the } \\
\text { puerperium }\end{array}$ & 671 \\
\hline Allergic rhinitis & 477 \\
\hline Allergic coinjunctivitis & $372.05,372.14$ \\
\hline Atopic dermatitia & 691.8 \\
\hline
\end{tabular}


Table 2. Demographic data

\begin{tabular}{|c|c|c|c|}
\hline & Asthma $(\mathrm{n}=3,231)$ & non-asthma $(\mathrm{n}=51,826)$ & p-value \\
\hline $\begin{array}{l}\text { mother's age } \\
\text { less than } 35 \mathrm{y} / \mathrm{o} \\
\text { equal or more than } 35 \mathrm{y} / \mathrm{o}\end{array}$ & $\begin{array}{c}2,625(81.24 \%) \\
606(18.76 \%)\end{array}$ & $\begin{array}{r}42,382(81.78 \%) \\
9,444(18.22 \%)\end{array}$ & 0.457 \\
\hline $\begin{array}{l}\text { Urban } \\
\text { Country }\end{array}$ & $\begin{array}{c}2,492(77.13 \%) \\
739(22.87 \%)\end{array}$ & $\begin{array}{l}39,621(76.45 \%) \\
12,205(23.55 \%)\end{array}$ & 0.378 \\
\hline $\begin{array}{l}\text { Socioeconomic status } \\
\text { Middle to high } \\
\text { Low }\end{array}$ & $\begin{array}{c}2,682(83.01 \%) \\
549(16.99 \%)\end{array}$ & $\begin{array}{r}42,761(82.51 \%) \\
9,065(17.49 \%)\end{array}$ & 0.468 \\
\hline $\begin{array}{l}\text { Vaginal delivery } \\
\text { Cesarean delivery }\end{array}$ & $\begin{array}{l}1,923(59.52 \%) \\
1,308(40.48 \%)\end{array}$ & $\begin{array}{l}33,128(63.92 \%) \\
18,698(36.08 \%)\end{array}$ & 0.000 \\
\hline $\begin{array}{l}\text { Medical co-morbidities } \\
\text { yes } \\
\text { no }\end{array}$ & $\begin{array}{c}132(4.09 \%) \\
3,099(95.91 \%)\end{array}$ & $\begin{array}{c}1,407(2.71 \%) \\
50,419(97.29 \%)\end{array}$ & 0.000 \\
\hline $\begin{array}{l}\text { Pregnancy characteristics } \\
\text { yes } \\
\text { no }\end{array}$ & $\begin{array}{r}145(4.49 \%) \\
3,086(95.51 \%)\end{array}$ & $\begin{array}{l}1,933(3.73 \%) \\
49,893(96.27 \%)\end{array}$ & 0.028 \\
\hline $\begin{array}{l}\text { Delivery complications } \\
\text { yes } \\
\text { no }\end{array}$ & $\begin{array}{l}1,021(31.60 \%) \\
2,210(68.40 \%)\end{array}$ & $\begin{array}{l}15,040(29.02 \%) \\
36,786(70.98 \%)\end{array}$ & 0.002 \\
\hline $\begin{array}{l}\text { TE } \\
\text { yes } \\
\text { no }\end{array}$ & $\begin{array}{c}8(2.47 \% \text { \%o } \\
3,223(997.53 \% \text { \% })\end{array}$ & $\begin{array}{c}37(0.71 \%) \\
51,789(999.29 \%)\end{array}$ & 0.001 \\
\hline $\begin{array}{l}\text { Venous complications } \\
\text { yes } \\
\text { no }\end{array}$ & $\begin{array}{c}23(7.12 \% \text { \% }) \\
3,208(992.88 \% \text { \% }\end{array}$ & $\begin{array}{c}355(6.85 \%) \\
51,471(993.15 \%)\end{array}$ & 0.858 \\
\hline
\end{tabular}


Table 3: Rate of TE in different asthma conditions

\begin{tabular}{|l|l|l|l|l|l|}
\hline group & VET rate (case/total, \%o) & p-value & Odds ratio & 95\% CI & adjust p-value* \\
\hline control & $37 / 51,826(0.71 \%$ ) & & & & \\
\hline group1 & $4 / 2,018(1.98 \%$ o $)$ & 0.043 & 2.78 & $0.99 \sim 7.81$ & 0.142 \\
\hline group2 & $3 / 775(3.97 \%$ o & $\mathbf{0 . 0 0 2}$ & $\mathbf{5 . 4 4}$ & $\mathbf{1 . 6 7 ~ 1 7 . 6 8}$ & $\mathbf{0 . 0 1 3}$ \\
\hline group3 & $1 / 438(2.28 \%)$ & 0.255 & 3.20 & $0.43 \sim 23.39$ & 0.407 \\
\hline
\end{tabular}

Adjusted p-value: adjusted for medical comorbidities, pregnancy characteristics, delivery complications, urbanization, jobs, mother's age and birth type 
Tabel 4: Rate of venous complications in pregnancy and puerperium in different asthma conditions

\begin{tabular}{|l|l|l|l|l|l|}
\hline group & complication rate (case/total, \%o) & p-value & Odds ratio & $95 \%$ CI & $\begin{array}{l}\text { Adjusted } \\
\text { p-value }\end{array}$ \\
\hline control & $355 / 51,826(6.85 \%)$ & & & & \\
\hline group1 & $12 / 2,018(5.94 \%$ ) & 0.628 & 0.87 & $0.49 \sim 1.54$ & 0.526 \\
\hline group2 & $4 / 775(5.16 \%)$ & 0.571 & 0.75 & $0.28 \sim 2.02$ & 0.525 \\
\hline group3 & $7 / 438(15.98 \%$ ) & $\mathbf{0 . 0 2 2}$ & $\mathbf{2 . 3 6}$ & $1.11 \sim 5.01$ & $\mathbf{0 . 0 4 3}$ \\
\hline
\end{tabular}

Adjusted p-value: adjusted for medical comorbidities, pregnancy characteristics, delivery complications, urbanization, jobs, mother's age and birth type 
Table 5: Rate of TE in different asthma conditions in CS subjects with different asthma conditions

\begin{tabular}{|l|l|l|l|l|l|}
\hline group & complication rate (case/total, \%o) & p-value & Odds ratio & $95 \%$ CI & \\
\hline control & $23 / 18,698(1.23 \%)$ & & & & \\
\hline group1 & $3 / 797(3.76 \%$ ) & 0.055 & 3.07 & $0.92 \sim 10.24$ & 0.155 \\
\hline group2 & $2 / 310(6.45 \%)$ & $\mathbf{0 . 0 1 2}$ & $\mathbf{5 . 2 7}$ & $\mathbf{1 . 2 4} \mathbf{2 2 . 4 6}$ & $\mathbf{0 . 0 4 6}$ \\
\hline group3 & $1 / 201(4.97 \%$ ) & 0.138 & 4.06 & $0.55 \sim 30.21$ & 0.250 \\
\hline
\end{tabular}

Adjusted p-value: adjusted for medical comorbidities, pregnancy characteristics, delivery complications, urbanization, jobs, mother's age and birth type 
Figure 1. Flow chart

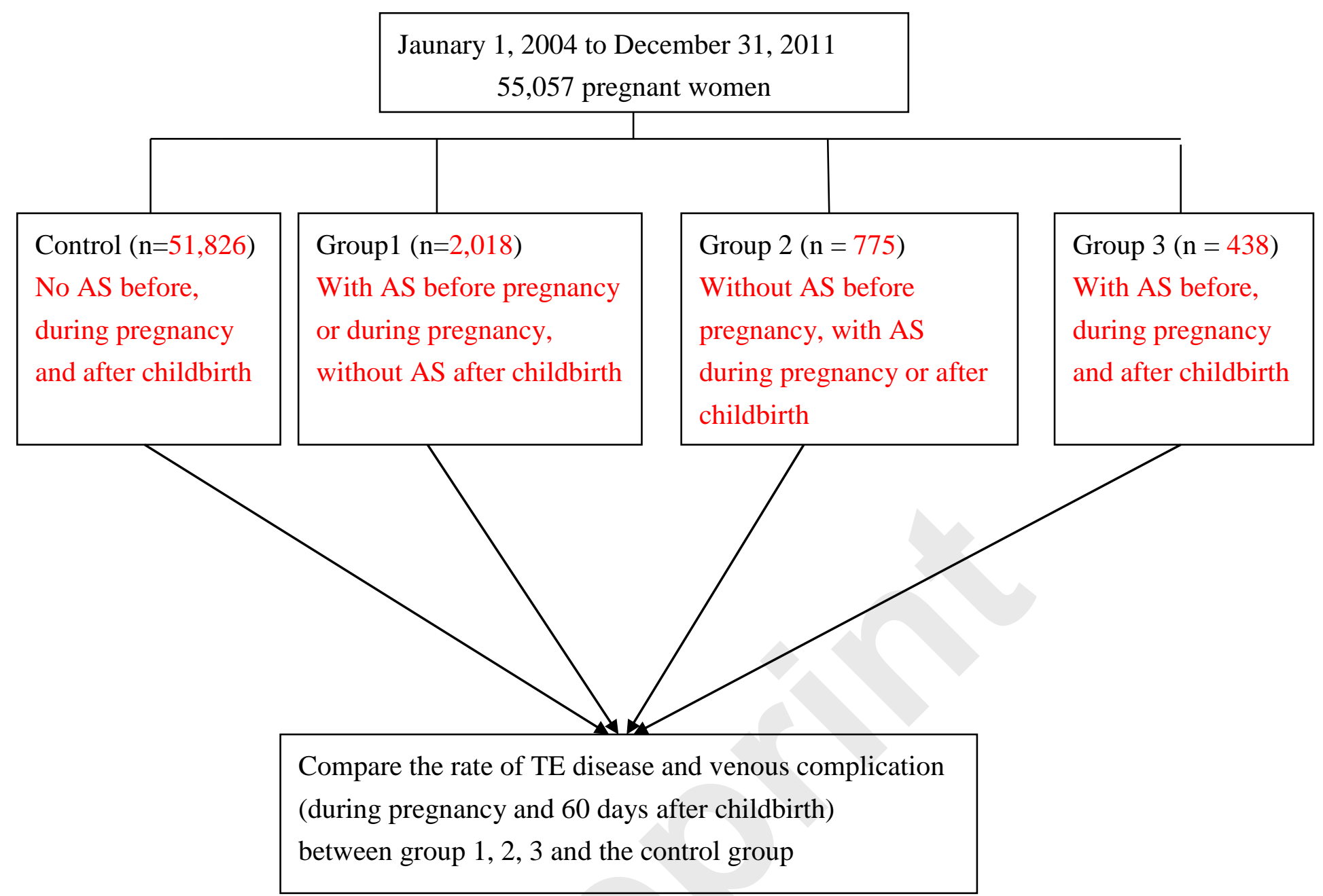


Table 1. ICD-9-CM codes used in this study.

\begin{tabular}{|c|c|}
\hline Disease & ICD-9-CM code \\
\hline Asthma & 493 \\
\hline \multicolumn{2}{|l|}{ Thromboembolism } \\
\hline TE of intracranial venous sinuses & 325 \\
\hline Pulmonary embolism and infarction & 415.1 \\
\hline TE of arteries, arterioles and capillaries & 444,445 \\
\hline Venous TE & $451,452,453$ \\
\hline Venous TE in pregnancy and puerperium & $671.2 \sim 671.5$ \\
\hline Obstetrical pulmonary embolism & $673.2,673.3,673.8$ \\
\hline \multicolumn{2}{|l|}{ Medical comorbidities } \\
\hline Type I DM with nephropathy & $250.41,250.43$ \\
\hline Chronic heart disease & $393-398,424-429$ \\
\hline Systemic lupus erythematosus & $695.4,710.0$ \\
\hline Inflammatory bowel disease & 555,556 \\
\hline Overweight and besity & 278 \\
\hline Thrombophilia & $286.53,289.81,289.82$ \\
\hline Nephrotic syndrome & 581 \\
\hline Tuberculosis & 010 018 \\
\hline Inflammatory polyarthropathy & 716.59 \\
\hline \multicolumn{2}{|l|}{ Pregnancy characteristics } \\
\hline Multiple gestation & 651 \\
\hline Preeclampsia & $642.4 \sim 642.7$ \\
\hline Pregnancy-related hypertension & $642.0 \sim 642.3,642.9$ \\
\hline \multicolumn{2}{|l|}{ Delivery complications } \\
\hline Early or threatened labor & 644 \\
\hline Postpartum hemorrhage & 666,667 \\
\hline Prolong labor & 662 \\
\hline Infection ( 7 days before $\sim 60$ days after & 590.1, 590.8, 590.9, 595.0 595.4, \\
\hline childbirth) & $595.9,647,658.4,659.2,659.3$ \\
\hline & $670.0,672,675,5990,486,998.59$ \\
\hline $\begin{array}{l}\text { Venous complications in pregnancy and the } \\
\text { puerperium }\end{array}$ & 671 \\
\hline Allergic rhinitis & 477 \\
\hline Allergic coinjunctivitis & $372.05,372.14$ \\
\hline Atopic dermatitia & 691.8 \\
\hline
\end{tabular}


Table 2. Demographic data

\begin{tabular}{|c|c|c|c|}
\hline & Asthma $(\mathrm{n}=3,231)$ & non-asthma $(\mathrm{n}=51,826)$ & p-value \\
\hline $\begin{array}{l}\text { mother's age } \\
\text { less than } 35 \mathrm{y} / \mathrm{o} \\
\text { equal or more than } 35 \mathrm{y} / \mathrm{o}\end{array}$ & $\begin{array}{c}2,625(81.24 \%) \\
606(18.76 \%)\end{array}$ & $\begin{array}{r}42,382(81.78 \%) \\
9,444(18.22 \%)\end{array}$ & 0.457 \\
\hline $\begin{array}{l}\text { Urban } \\
\text { Country }\end{array}$ & $\begin{array}{c}2,492(77.13 \%) \\
739(22.87 \%)\end{array}$ & $\begin{array}{l}39,621(76.45 \%) \\
12,205(23.55 \%)\end{array}$ & 0.378 \\
\hline $\begin{array}{l}\text { Socioeconomic status } \\
\text { Middle to high } \\
\text { Low }\end{array}$ & $\begin{array}{c}2,682(83.01 \%) \\
549(16.99 \%)\end{array}$ & $\begin{array}{r}42,761(82.51 \%) \\
9,065(17.49 \%)\end{array}$ & 0.468 \\
\hline $\begin{array}{l}\text { Vaginal delivery } \\
\text { Cesarean delivery }\end{array}$ & $\begin{array}{l}1,923(59.52 \%) \\
1,308(40.48 \%)\end{array}$ & $\begin{array}{l}33,128(63.92 \%) \\
18,698(36.08 \%)\end{array}$ & 0.000 \\
\hline $\begin{array}{l}\text { Medical co-morbidities } \\
\text { yes } \\
\text { no }\end{array}$ & $\begin{array}{c}132(4.09 \%) \\
3,099(95.91 \%)\end{array}$ & $\begin{array}{c}1,407(2.71 \%) \\
50,419(97.29 \%)\end{array}$ & 0.000 \\
\hline $\begin{array}{l}\text { Pregnancy characteristics } \\
\text { yes } \\
\text { no }\end{array}$ & $\begin{array}{r}145(4.49 \%) \\
3,086(95.51 \%)\end{array}$ & $\begin{array}{l}1,933(3.73 \%) \\
49,893(96.27 \%)\end{array}$ & 0.028 \\
\hline $\begin{array}{l}\text { Delivery complications } \\
\text { yes } \\
\text { no }\end{array}$ & $\begin{array}{l}1,021(31.60 \%) \\
2,210(68.40 \%)\end{array}$ & $\begin{array}{l}15,040(29.02 \%) \\
36,786(70.98 \%)\end{array}$ & 0.002 \\
\hline $\begin{array}{l}\text { TE } \\
\text { yes } \\
\text { no }\end{array}$ & $\begin{array}{c}8(2.47 \%) \\
3,223(997.53 \% \text { \% })\end{array}$ & $\begin{array}{c}37(0.71 \%) \\
51,789(999.29 \%)\end{array}$ & 0.001 \\
\hline $\begin{array}{l}\text { Venous complications } \\
\text { yes } \\
\text { no }\end{array}$ & $\begin{array}{c}23(7.12 \% \text { \% }) \\
3,208(992.88 \% \text { \% }\end{array}$ & $\begin{array}{c}355(6.85 \%) \\
51,471(993.15 \%)\end{array}$ & 0.858 \\
\hline
\end{tabular}


Table 3: Rate of TE in different asthma conditions

\begin{tabular}{|l|l|l|l|l|l|}
\hline group & VET rate $($ case/total, \%o) & p-value & Odds ratio & 95\% CI & adjust p-value* \\
\hline control & $37 / 51,826(0.71 \%$ ) & & & & \\
\hline group1 & $4 / 2,018(1.98 \%)$ & 0.043 & 2.78 & $0.99 \sim 7.81$ & 0.142 \\
\hline group2 & $3 / 775(3.97 \%$ ) & $\mathbf{0 . 0 0 2}$ & $\mathbf{5 . 4 4}$ & $\mathbf{1 . 6 7 ~ 1 7 . 6 8}$ & $\mathbf{0 . 0 1 3}$ \\
\hline group3 & $1 / 438(2.28 \%)$ & 0.255 & 3.20 & $0.43 \sim 23.39$ & 0.407 \\
\hline
\end{tabular}

Adjusted p-value: adjusted for medical comorbidities, pregnancy characteristics, delivery complications, urbanization, jobs, mother's age and birth type 
Tabel 4: Rate of venous complications in pregnancy and puerperium in different asthma conditions

\begin{tabular}{|l|l|l|l|l|l|}
\hline group & complication rate (case/total, \%o) & p-value & Odds ratio & $95 \%$ CI & $\begin{array}{l}\text { Adjusted } \\
\text { p-value }\end{array}$ \\
\hline control & $355 / 51,826(6.85 \%)$ & & & & \\
\hline group1 & $12 / 2,018(5.94 \%$ ) & 0.628 & 0.87 & $0.49 \sim 1.54$ & 0.526 \\
\hline group2 & $4 / 775(5.16 \%)$ & 0.571 & 0.75 & $0.28 \sim 2.02$ & 0.525 \\
\hline group3 & $7 / 438(15.98 \%$ o $)$ & $\mathbf{0 . 0 2 2}$ & $\mathbf{2 . 3 6}$ & $1.11 \sim 5.01$ & $\mathbf{0 . 0 4 3}$ \\
\hline
\end{tabular}

Adjusted p-value: adjusted for medical comorbidities, pregnancy characteristics, delivery complications, urbanization, jobs, mother's age and birth type 
Table 5: Rate of TE in different asthma conditions in CS subjects with different asthma conditions

\begin{tabular}{|l|l|l|l|l|l|}
\hline group & complication rate (case/total, \%o) & p-value & Odds ratio & $95 \%$ CI & \\
\hline control & $23 / 18,698(1.23 \%)$ & & & & \\
\hline group1 & $3 / 797(3.76 \%$ ) & 0.055 & 3.07 & $0.92 \sim 10.24$ & 0.155 \\
\hline group2 & $2 / 310(6.45 \%)$ & $\mathbf{0 . 0 1 2}$ & $\mathbf{5 . 2 7}$ & $\mathbf{1 . 2 4 ~ 2 2 . 4 6}$ & $\mathbf{0 . 0 4 6}$ \\
\hline group3 & $1 / 201(4.97 \%$ ) & 0.138 & 4.06 & $0.55 \sim 30.21$ & 0.250 \\
\hline
\end{tabular}

Adjusted p-value: adjusted for medical comorbidities, pregnancy characteristics, delivery complications, urbanization, jobs, mother's age and birth type 